\title{
El consumo persistente de cannabis se asocia a un leve deterioro neuropsicológico
}

Persistent cannabis consumption is associated with mild neuropsychological impairment

Meiere MH y cols. PNAS 2012 Octubre 2;109(40):E2657-64.

\section{Objetivo}

Establecer la relación existente entre el nivel de consumo de cannabis y la performance neuropsicológica (PN).

\section{Diseño y población}

Cohorte prospectiva (Dunedin Multidisciplinary Health and Development Study) que comprendió 1.037 nacimientos consecutivos entre el 1ero de Abril de 1972 y el 31 de Marzo de 1973 en Dunedin, Nueva Zelanda. Fue establecida a los tres años, comprendiendo $91 \%$ de los nacimientos (52\% varones). Se midió la PN pre y post-exposición, en puntos de coeficiente intelectual $(\mathrm{Cl})$, mediante pruebas neuropsicológicas estandarizadas acordes a la edad del paciente.

\section{Evaluación de los factores de riesgo y medición de resulta-} dos principales

Se evaluó la PN previa a la exposición al cannabis $(7,9,11$ y 13 años) y posterior (a los 38 años). El consumo de cannabis fue investigado mediante el Diagnostic Interview Schedule a los
18, 21, 26, 32 y 38 años. En base a los resultados en cada instancia de entrevista, se clasifico a los pacientes según sus hábitos de consumo (nunca consumió; consumo no regular; consumo regular; dependencia).

\section{Resultados Principales}

Se calculó la diferencia de puntos de $\mathrm{Cl}$ entre la etapa preexposición y la etapa post-exposición para cada paciente y se lo analizó en base a cómo fue clasificado según su hábito de consumo. Se observó una relación directa entre caída del $\mathrm{Cl}$ y mayor consumo de cannabis (Tabla 1). Ésta tendencia se mantiene en los diferentes dominios neuropsicológicos (funciones ejecutivas, memoria, velocidad de procesamiento, comprensión verbal, razonamiento perceptual). Por otro lado, en pacientes con consumo precoz y severo (al menos un diagnóstico de dependencia previo a los 18 años y más de tres a los 38 ), el deterioro neuropsicológico tras 20 años de consumo sería mayor que en aquellos con más de tres diagnósticos a los 38 años, pero sin dependencia antes de los $18(p=0,02)$.

Tabla 1: $\mathrm{Cl}$ antes y después del consumo de cannabis

\begin{tabular}{l|c|c|c|c}
\multicolumn{1}{c|}{ Tipo de consumo } & N & CI Pre-exposición & Cl Post-exposición & Dif. de Cl expresado en DS \\
\hline Nunca consumió & 242 & 99,84 & 100,64 & 0,05 \\
\hline$>3$ diagnósticos de dependencia & 38 & 99,68 & 93,93 & $-0,38$ \\
\hline$>3$ diagnósticos de uso regular & 41 & 96,00 & 90,77 & $-0,35$ \\
\hline
\end{tabular}

\section{Conclusión}

El uso persistente de cannabis a lo largo de más de 20 años está asociado a deterioro neuropsicológico, siendo el deterioro mayor, mientras mayor sea el consumo.

\section{Comentario}

La marihuana se convertido en una de las drogas ilegales más consumida en el mundo'. Muchos de sus efectos en el corto plazo han sido estudiados y se posee evidencia de buena calidad para poder aconsejar a los pacientes sobre su consumo ${ }^{2}$. Sin embargo la información de los efectos a largo plazo, especialmente a nivel cognitivo, provenía de estudios cuya calidad metodológica era limitada ${ }^{3-5}$. Ésta cohorte prospectiva presenta un seguimiento a 20 años de los pacientes, lo cual se acerca bastante a lo puede considerarse ideal a la hora de evaluar repercusiones en el largo plazo, especialmente teniendo en cuenta las limitantes éticas para desarrollar ensayos clínicos aleatorizados que puedan demostrar una clara relación causa-efecto.

\section{Conclusiones del comentador}

Las conclusiones de los autores nos plantean que el consumo persistente se asocia a una caída en el puntaje de $\mathrm{Cl}$ a lo largo de 20 años. En el grupo de mayor consumo esa caída llega a 8 puntos de $\mathrm{Cl}$, lo que representa $-0,38$ de DS aproximándose a los 90 puntos.

Si se tiene en cuenta que el $95 \%$ de la población está dentro de +/- 2DS (100 +/- $30 \mathrm{Cl}$ ) esta caída de 8 puntos totales no impresiona como un gran deterioro en términos absolutos.

Santiago Esteban [ Servicio de Medicina Familiar y Comunitaria del Hospital Italiano de Buenos Aires santiago.esteban@hospitalitaliano.org.ar ]

Esteban S. El consumo persistente de cannabis se asocia a un leve deterioro neuropsicológico. Evid Act Pract Ambul . Ene-Mar. 2013;(16)1:9. Comentado de: Meier MH, et al. Persistent cannabis users show neuropsychological decline from childhood to midlife. Proc Natl Acad Sci U S A. 2012 Oct 2;109(40):E2657-64. PMID:22927402.

\section{Referencias}

1. Murray RM, Morrison PD, Henquet C, Di Forti M. Cannabis, the mind and society: The hash realities. Nat Rev Neurosci 2007;8:885-895.

2. Asbridge M, Hayden JA, Cartwright JL. Acute cannabis consumption and motor vehicle collision risk: systematic review of observational studies and meta-analysis. BMJ. 2012 Feb 9;344:e536. doi: 10.1136/bmj.e536.

3. Solowij N, Battisti R. The chronic effects of cannabis on memory in humans: A review. Curr Drug Abuse Rev 2008;1:81-98.

4. Solowij N, et al. Marijuana Treatment Project Research Group. Cognitive functioning of long-term heavy cannabis users seeking treatment. JAMA 2002;287:1123-1131.

5. Pope HG, Jr., Yurgelun-Todd D. The residual cognitive effects of heavy marijuana use in college students. JAMA 1996;275:521-527. 